\title{
An estimate for the measure theoretic entropy of geodesic flows
}

\author{
WERNER BALLMANN† \\ Universität Zürich, 8001, Switzerland \\ MACIEJ P. WOJTKOWSKI† \\ University of Arizona, Tucson AZ 85721, USA \\ (Received 27 July 1987 and revised 13 May 1988)
}

Abstract. A new proof and a generalization of the Osserman-Sarnak estimate for the measure theoretic entropy of geodesic flows is presented.

In this note we discuss an estimate of Osserman and Sarnak [O-S] for the measure theoretic entropy of geodesic flows. Let $M$ be a compact Riemannian manifold with nonpositive sectional curvature and denote by $R$ the curvature tensor of $M$. Then for all $p \in M$ and all unit vectors $v \in T_{p} M, K(v):=R(\cdot, v) v$ is a nonpositive symmetric operator on $T_{p} M$. The estimate we will obtain is

$$
h_{\mu} \geq \int_{S M} \operatorname{tr} \sqrt{-K(v)} d \mu(v),
$$

where $h_{\mu}$ is the measure theoretic entropy of the geodesic flow of $M, S M$ is the unit tangent bundle of $M$ and $\mu$ denotes the normalized Liouville measure on $S M$. Moreover,

$$
h_{\mu}=\int_{S M} \operatorname{tr} \sqrt{-K(v)} d \mu(v) \quad \text { iff } M \text { is locally symmetric. }
$$

The results, (1) and (2), were obtained by Osserman and Sarnak in the case that the sectional curvature of $M$ is strictly negative. We refer the reader to [O-S] for a formulation and comparison of the estimates which preceded (1) and respective references.

The measure theoretic entropy of a measure preserving flow is an asymptotic quantity associated with the flow. In the case under consideration Pesin [P2] showed that $h_{\mu}$ is given by the average of the mean curvatures of the horospheres. The significance of (1) lies in the fact that it estimates the asymptotic quantity $h_{\mu}$ by an average of local quantities associated to $M$.

Our proof of (1) and (2) is a simplification of the proof of Osserman and Sarnak and it works under the weaker assumption of nonpositive sectional curvature. Note that this is the weakest assumption under which (1) and (2) make sense. An important

† Partially supported by NSF Grants DMS-8503742 and DMS-8601897. 
feature of our approach is that we estimate the exponential rate of growth of certain volumes along individual but typical geodesics. In the course of the discussion we will also obtain formulas of Pesin [P2] and Freire and Mañe [F-M] for $h_{\mu}$.

\section{Multiplicative ergodic theorem and entropy formula}

In this section we will state the Oseledets Multiplicative Ergodic Theorem [Os] in the form in which we will use it. Let $\left\{g^{t}\right\}$ be a smooth flow without fixed points on a compact manifold $X$ preserving a smooth probability measure $\mu$ on $X$. We are interested in exponential growth rates associated with $d g^{\prime}$.

Let $u(x)=\left.(d / d t) g^{t} x\right|_{t=0}$ be the velocity vector of the flow. Then $d g^{t} u(x)=u\left(g^{\prime} x\right)$ and therefore it is natural to consider, instead of $d g^{t}$, the quotient linear operators

$$
L_{x}^{\prime}: Q_{x} \rightarrow Q_{g} t_{x}
$$

where $Q_{x}$ is the quotient of $T_{x} X$ by the 1-dimensional subspace spanned by $u(x)$. These operators contain all the information about the exponential growth and decay under $d g^{t}$ which we need.

Assume $\|\cdot\|$ is a Riemannian norm on the bundle $Q \rightarrow X$. Then for almost all $x \in X$, the limit

$$
\lim _{t \rightarrow \pm \infty} \frac{1}{|t|} \ln \left\|L_{x}^{t} w\right\|:=\chi^{ \pm}(x, w)
$$

exists for all $x \in Q_{x} \backslash\{0\}$ and it is called a Lyapunov exponent. Lyapunov exponents are independent of the choice of $\|\cdot\|$. We define $\chi^{ \pm}(x, 0):=-\infty$.

For almost every $x \in X$, we obtain subspaces

$$
\begin{aligned}
& E^{u}(x)=\left\{w \in Q_{x} \mid \chi^{-}(x, w)<0\right\}, \\
& E^{s}(x)=\left\{w \in Q_{x} \mid \chi^{+}(x, w)<0\right\}, \\
& E^{0}(x)=\left\{w \in Q_{x} \mid \chi^{-}(x, w) \leq 0 \text { and } \chi^{+}(x, w) \leq 0\right\} .
\end{aligned}
$$

By the Oseledets Theorem for almost every $x \in X$ these subspaces are linearly independent and they span $Q_{x}$. Furthermore for any subspace $E$ such that $E^{u}(x) \subset$ $E \subset E^{u}(x) \oplus E^{0}(x)$ the exponential rates of volume growth in $E$, that is the limits

$$
\lim _{t \rightarrow \pm \infty} \frac{1}{|t|} \ln \left|\operatorname{det}\left(\left.L_{x}^{\prime}\right|_{E}\right)\right|= \pm \chi(x)
$$

exist and they differ by the sign alone in the future and in the past (cf. [F-M]). Also $\chi(x)$ does not depend on the choice of $E$ as above and $\chi(x)>0$ unless $E^{u}(x)=\{0\}$. In fact $\chi(x)$ is equal to the sum of the positive Lyapunov exponents taken with multiplicities but this will not be used in the sequel. By the Pesin Entropy Formula [P1] the metric entropy $h_{\mu}$ of the flow $\left\{g^{t}\right\}$ with respect to the measure $\mu$ is equal to the average exponential rate of volume growth, that is

$$
h_{\mu}=\int_{X} \chi(x) d \mu(x)
$$

\section{The geodesic flow}

Let $M$ be a compact Riemannian manifold of dimension $n$ and of nonpositive sectional curvature. By $S M$ we denote the unit tangent bundle of $M$ and by $\gamma_{v}$, 
$v \in S M$, the geodesic with initial velocity $v$. The geodesic flow $g^{\mathbf{t}}: S M \rightarrow S M, t \in \mathbb{R}$, is defined by $g^{t} v=\dot{\gamma}_{v}(t)$. The flow $g^{t}$ preserves the normalized Liouville measure $\mu$ on $S M$.

Let $v \in S M$ and $p \in M$ be the footpoint of $v$. Then $T_{v} S M$ is naturally isomorphic to $T_{p} M \oplus V(v)$, where $V(v) \subset T_{p} M$ is the orthogonal complement of $v$ in $T_{p} M$. With this identification the differential $d g^{t}$ of $g^{t}$ at $v$ is given by

$$
d g^{t}(x, y)=\left(J(t), J^{\prime}(t)\right)
$$

where $J$ is the Jacobi field along $\gamma_{v}$ with the initial condition $J(0)=x, J^{\prime}(0)=y$. Since $d g^{\prime}(V(v) \oplus V(v))=V\left(g^{\prime} v\right) \oplus V\left(g^{\prime} v\right)$, the restriction of $d g^{\prime}$ to $V(v) \oplus V(v)$ is a canonical representation of the quotient $L^{\prime}$ discussed in $\S 1$. This corresponds to considering Jacobi fields perpendicular to $\gamma_{v}$. Recall that Jacobi fields satisfy the Jacobi equation, namely

$$
J^{\prime \prime}+K\left(g^{t} v\right) J=0,
$$

where $K(v): V(v) \rightarrow V(v)$ is defined by $K(v) x=R(x, v) v$ and $R$ denotes the curvature tensor. The operator $K(v)$ is symmetric and since the sectional curvature is nonpositive it is also nonpositive. Noted that this implies that $\|J(t)\|^{2}$ is a convex function of $t$. Indeed

$$
\langle J, J\rangle^{\prime \prime}=2\left\langle J^{\prime \prime}, J\right\rangle+2\left\langle J^{\prime}, J^{\prime}\right\rangle=-2\langle K J, J\rangle+2\left\langle J^{\prime}, J^{\prime}\right\rangle \geq 0 .
$$

It is useful to introduce the symplectic form $\omega$ on $V(v) \oplus V(v)$ defined by

$$
\omega\left(\left(x_{1}, y_{1}\right),\left(x_{2}, y_{2}\right)\right)=\left\langle x_{1}, y_{2}\right\rangle-\left\langle x_{2}, y_{1}\right\rangle \text {. }
$$

By the force of (5) this form is preserved under $d g^{t}$.

We denote by $H(v)$ the linear subspace of $V(v) \oplus V(v)$ defined by the condition that $(x, y) \in H(v)$ if the Jacobi field $J$ along $\gamma_{v}$ determined by $J(0)=x, J^{\prime}(0)=y$ is nondecreasing in norm for all $t$. By convexity we have

$$
(x, y) \in H(v) \quad \text { iff }\|J(t)\| \quad \text { remains bounded for } t \leq 0 \text {. }
$$

It is well known that $H(v)$ is the graph of the second fundamental form $U(v): V(v) \rightarrow$ $V(v)$ of the horosphere determined by $-v$. The operator $U(v)$ is symmetric nonpositive and since $d g^{\prime} H(v)=H\left(g^{t} v\right)$ it satisfies by the force of (5) the Riccati equation

$$
U^{\prime}+U^{2}+K\left(g^{\prime} v\right)=0,
$$

where $U^{\prime}$ denotes the covariant derivative of $U\left(g^{\prime} v\right)$ along $\gamma_{v}$. Note that $H(v)$ is a Lagrangian subspace of $V(v) \oplus V(v)$ since $U(v)$ is symmetric.

For notational simplicity we will sometimes suppress the dependence on $v$.

Proposition 2.1. $E^{u} \subset H \subset E^{u} \oplus E^{0}$.

Proof. We will first establish the general fact that $E^{u}$ and $E^{u} \oplus E^{0}$ are skeworthogonal complements of each other. Indeed, if $w_{1} \in E^{u}$ and $w_{2} \in E^{u} \oplus E^{0}$, then since $\|\omega\|=1$ we have

$$
\lim _{i \rightarrow-\infty} \frac{1}{|l|} \ln \left|\omega\left(d g^{t} w_{1}, d g^{t} w_{2}\right)\right|<0 .
$$

The symplectic form $\omega$ is preserved by $d g^{t}$, so that $\omega\left(d g^{\prime} w_{1}, d g^{\prime} w_{2}\right)$ is constant in $t$, and we conclude that $\omega\left(w_{1}, w_{2}\right)=0$. A skeworthogonal complement of a subspace 
has the complementary dimension, hence

$$
\operatorname{dim} E^{u}+\operatorname{dim} E^{u} \oplus E^{0} \leq 2(n-1) .
$$

Similarly

$$
\operatorname{dim} E^{s}+\operatorname{dim} E^{s} \oplus E^{0} \leq 2(n-1) .
$$

But because $\operatorname{dim} E^{s}+\operatorname{dim} E^{u}+\operatorname{dim} E^{0}=2(n-1)$ we conclude that there must be equalities and hence $E^{u} \oplus E^{0}$ is the skeworthogonal complement of $E^{u}$.

$E^{u} \subset H$ by (6). It follows that the skeworthogonal complement of $E^{u}$, which is $E^{u} \oplus E^{0}$, must contain the skeworthogonal complement of $H$, which is $H$ itself because $H$ is a Lagrangian subspace.

In the case of negative sectional curvatures $E^{u}(v)=H(v)$. When the sectional curvature is allowed to vanish $E^{u}(v)$ may be much smaller than $H(v)$. But $H(v)$ is a more geometric object than $E^{u}(v)$ is. In particular $H(v)$ is well defined for every $v \in S M$ and $E^{u}(v)$ is defined only almost everywhere.

By (3) and (4) and the above Proposition we can express the metric entropy of $\left\{g^{t}\right\}$ by the exponential rates of volume growth in $H\left(g^{t} v\right)$. However, to be able to treat the case of nonpositive sectional curvature as opposed to negative sectional curvature we have to work with a subspace of $H(v)$. Let $V_{0}(v)$ be the orthogonal complement of $\operatorname{ker} U(v)$ in $V(v)$.

Lemma 2.2. (i) $x \in \operatorname{ker} U(v)$ iff the Jacobi field determined by $J(0)=x, J^{\prime}(0)=0$ satisfies $J^{\prime}(t)=0$ for all $t \leq 0$.

(ii) $K(v)=0$ on $\operatorname{ker} U(v)$ and $U(v)$ and $K(v)$ preserve $V_{0}(v)$.

Proof. Since $\|J(t)\|^{2}$ is convex and nondecreasing and $\left\langle J^{\prime}(0), J(0)\right\rangle=0$ we conclude that $\|J(t)\|=\|J(0)\|$ for $t \leq 0$. Thus we get for $t \leq 0$

$$
0=\frac{1}{2}(J, J\rangle^{\prime \prime}=\left\langle J^{\prime}, J\right\rangle^{\prime}=-\langle K J, J\rangle+\left\langle J^{\prime}, J^{\prime}\right\rangle .
$$

Since $K \leq 0$ we get $J^{\prime}=0$ and $K J=0$ for $t \leq 0$, and (i) follows immediately. Furthermore we see that $\operatorname{ker} U(v) \subset \operatorname{ker} K(v)$. Since $K(v)$ is symmetric we conclude that $K(v)$ preserves $V_{0}(v)$. This proves the Lemma.

It follows from the above Lemma that $\operatorname{dim} V_{0}\left(g^{t} v\right)$ is nondecreasing in $t$ and that $d g^{t}\left(V_{0}(v) \oplus V_{0}(v)\right) \subset V_{0}\left(g^{t} v\right) \oplus V_{0}\left(g^{t} v\right)$ for $t \geq 0$.

LEMMA 2.3. $\operatorname{dim} V_{0}\left(g^{t} v\right) \equiv$ constant in $t$ for $v$ from a $g^{t}$-invariant subset $\Omega \subset S M$ of full measure.

Proof. Any measurable function, which is nondecreasing along the orbits of a measurable flow preserving a probability measure, is equal on a set of full measure to a function constant on the orbits.

Lemma 2.2 implies that $V_{0}\left(g^{i} v\right)$ is the parallel translate of $V_{0}(v)$ along $\gamma_{v}$ for all $t \in \mathbb{R}$ and $v \in \Omega$.

Let us denote by $U_{0}(v)$ and $K_{0}(v)$ the restrictions of $U(v)$ and $K(v)$ to $V_{0}(v)$. We define

$$
H_{0}(v):=H(v) \cap\left(V_{0}(v) \oplus V_{0}(v)\right)=\operatorname{graph} U_{0}(v) .
$$

By Lemmas 2.2 and $2.3 d g^{\prime} H_{0}(v)=H_{0}\left(g^{\prime} v\right)$ for $v \in \Omega$. 
For $v \in \Omega$ a Jacobi field $J$ along $\gamma_{v}$ splits into $J=J_{1}+J_{2}$, where $J_{1}(t) \in \operatorname{ker} U\left(g^{t} v\right)$ and $J_{2}(t) \in V_{0}\left(g^{\prime} v\right)$ are Jacobi fields since $K\left(g^{\prime} v\right) \equiv 0$ on $\operatorname{ker} U\left(g^{\prime} v\right)$ and $\operatorname{ker} U\left(g^{\prime} v\right)$ is parallel along $\gamma_{v}$. We easily conclude that $E^{u}(v) \subset H_{0}(v)$ for $v \in \Omega$.

Note that by construction $U_{0}$ is an invertible operator.

3. Volume growth and entropy estimate

It is useful to consider different volume elements in $H_{0}(v)$. Since $H_{0}(v)=$ graph $U_{0}(v)$, we may identify $H_{0}(v)$ with $V_{0}(v)$ by the projection onto the first factor and thus write a scalar product in $H_{0}(v)$ as $\langle\mathrm{A}(\mathrm{v}) \cdot, \cdot\rangle$, where $A(v)$ is a positive operator on $V_{0}(v)$. For example the standard scalar product in $H_{0}(v)$ is given by $A(v)=I+U_{0}^{2}$. Given scalar products in $H_{0}\left(g^{t} v\right)$ let

$$
a(t)=\left|\operatorname{det} d g^{\prime}\right|_{H_{0}(v)} \mid
$$

be the Jacobian of $d g^{t}$ restricted to $H_{0}(v)$ with respect to the scalar products. A classical computation yields

$$
r(t):=\frac{d}{d t} \ln a(t)=\frac{1}{2} \operatorname{tr} A^{\prime} A^{-1}+\operatorname{tr} U_{0}
$$

Note that $a(t)$ is defined along a particular geodesic but $r$ is actually a function on $S M$ (defined almost everywhere).

Proposition 3.1.

$$
h_{\mu}=\int_{S M} \operatorname{tr}\left(U_{0}-K_{0} U_{0}\right)\left(I+U_{0}^{2}\right)^{-1} d \mu .
$$

Proof. Applying (9) to the standard scalar product we get by the Riccati equation (7)

$$
A^{\prime}=U_{0}^{\prime} U_{0}+U_{0} U_{0}^{\prime}=-2 U_{0}^{3}-K_{0} U_{0}-U_{0} K_{0}
$$

and

$$
r(t)=\operatorname{tr}\left(U_{0}-K_{0} U_{0}\right)\left(I+U_{0}^{2}\right)^{-1}
$$

Furthermore we have

$$
\chi(v)=\lim _{T \rightarrow+\infty} \frac{1}{T} \ln a(T)=\lim _{T \rightarrow+\infty} \frac{1}{T} \int_{0}^{T} r(t) d t
$$

and since $r$ is an integrable function on $S M$ we get by the Birkhoff Ergodic Theorem

$$
h_{\mu}=\int_{S M} \chi(v) d \mu(v)=\int_{S M} r d \mu .
$$

For the purpose of proving the above Proposition we could have worked as well with $H, U$ and $K$. Anyway, by our construction in $\S 2$, replacing $U_{0}$ and $K_{0}$ by $U$ and $K$ will not change the trace so that we obtain immediately

$$
h_{\mu}=\int_{S M} \operatorname{tr}(U-K U)\left(I+U^{2}\right)^{-1} d \mu .
$$

This is one of Pesin's formulas for the metric entropy of the geodesic flow, [P2]. It holds under the assumption that the metric has no conjugate points, if we define $U$ appropriately and our proof works in that case also, once we establish that $E^{u} \subset$ graph $U \subset E^{u} \oplus E^{0}$. 
Now we will consider three other scalar products, namely

1. $A=I$,

2. $A=U_{0}^{2}$,

3. $A=U_{0}$.

The scalar product $1(2)$ is the standard scalar product in $V_{0}$ transported to $H_{0}$ by the projection onto the first (second) factor. All three scalar products are smaller than the standard scalar product. The norm of a nonzero vector in $H_{0}$ is increasing under $d g^{\prime}$ for the scalar products 1 and 3 (but not necessarily so for the scalar product 2).

Let $a_{i}$ and $r_{i}, i=1,2,3$, be defined as in (8) and (9) for the respective scalar products $1,2,3$ and let $c_{i}$ be the ratio of the standard volume element in $H_{0}$ and the volume element determined by the $i$ th scalar product. By straightforward computations, using (9) and the Riccati equation (7) we get

$$
\begin{aligned}
& r_{1}=\operatorname{tr} U_{0}, \\
& r_{2}=\operatorname{tr}\left(-K_{0} U_{0}^{-1}\right), \\
& r_{3}=\frac{1}{2} \operatorname{tr}\left(U_{0}-K_{0} U_{0}^{-1}\right) .
\end{aligned}
$$

We have

$$
0<a_{i}(t)<a(t) c_{i}(0) \text { and } r_{i}(t)>0
$$

for all $t \in \mathbb{R}, i=1,2,3$. The first claim follows from the fact that the volume element defined by the $i$ th scalar product is smaller than the standard volume element. The second claim follows from $\operatorname{tr}\left(-K_{0} U_{0}^{-1}\right)=\operatorname{tr}\left(\sqrt{-K_{0}} U_{0}^{-1} \sqrt{-K_{0}}\right)>0$. It follows from (10) and (3) that

$$
\underset{T \rightarrow+\infty}{\limsup } \frac{1}{T} \int_{0}^{T} r_{i}(t) d t=\limsup _{T \rightarrow+\infty} \frac{1}{T} \ln a_{i}(T) \leq \lim _{T \rightarrow+\infty} \frac{1}{T} \ln a(T)=\chi(v),
$$

and

$$
\liminf _{T \rightarrow-\infty} \frac{1}{|T|} \int_{T}^{0} r_{i}(t) d t=-\limsup _{T \rightarrow-\infty} \frac{1}{|T|} \ln a_{i}(T) \geq-\lim _{T \rightarrow-\infty} \frac{1}{|T|} \ln a(T)=\chi(v) .
$$

Putting the inequalities together we obtain

$$
\underset{T \rightarrow+\infty}{\limsup } \frac{1}{T} \int_{0}^{T} r_{i}(t) d t \leq \chi(v) \leq \liminf _{T \rightarrow-\infty} \frac{1}{|T|} \int_{T}^{0} r_{i}(t) d t .
$$

Under the assumption that $r_{i}$ is integrable as a function on $S M$ we obtain from (11) by the Birkhoff Ergodic Theorem and the equality of time averages in the future and in the past that

$$
\int_{S M} r_{i} d \mu=\int_{S M} \chi(v) d \mu(v)=h_{\mu}
$$

Integrability of $r_{1}$ follows from the fact that the principal curvatures of horospheres are bounded, but we do not know how to establish the integrability of $r_{2}$ (and $r_{3}$ ) by geometric considerations. We will obtain the integrability of $r_{i}, i=1,2,3$, from (11) by a bit of Ergodic Theory.

LEMMA 3.2 Let $\left\{g^{\prime}\right\}$ be a measure preserving flow on a probability space $(X, \mu)$, and 
let $f: X \rightarrow \mathbb{R}$ be a measurable nonnegative function. If for almost every $x \in X$

$$
\limsup _{T \rightarrow+\infty} \frac{1}{T} \int_{0}^{T} f\left(g^{\prime} x\right) d t \leq k(x),
$$

where $k: X \rightarrow \mathbb{R}$ is a measurable function, then

$$
\int_{X} f(x) d \mu(x) \leq \int_{X} k(x) d \mu(x) .
$$

Proof. Let

$$
f_{N}(x)= \begin{cases}f(x) & \text { if } f(x) \leq N \\ N & \text { if } f(x) \geq N .\end{cases}
$$

We have $0 \leq f_{N} \leq f$ and $f_{N} \in L^{1}(X, \mu)$. By the Birkhoff Ergodic Theorem, for almost all $x$

$$
\lim _{T \rightarrow+\infty} \frac{1}{T} \int_{0}^{T} f_{N}\left(g^{t} x\right) d t=f_{N}^{*}(x)
$$

and

$$
\int_{X} f_{N}^{*}(x) d \mu(x)=\int_{X} f_{N}(x) d \mu(x) .
$$

By assumption $f_{N}^{*}(x) \leq k(x)$ and so

$$
\int_{X} f_{N}(x) d \mu(x) \leq \int_{X} k(x) d \mu(x) .
$$

Hence

$$
\int_{X} f(x) d \mu(x) \leq \int_{X} k(x) d \mu(x) .
$$

Applying the above Lemma we get

Proposition 3.3

$$
h_{\mu}=\int_{S M} \operatorname{tr} U_{0} d \mu(v)=\int_{S M} \operatorname{tr}\left(-K_{0} U_{0}^{-1}\right) d \mu(v) .
$$

The first formula was obtained by Pesin [P2] in the case of a metric without conjugate points, see also [F-M]. One formula would follow immediately from the other if we would have the integrability of $\ln \operatorname{det} U_{0}$. Indeed $\left(\ln \operatorname{det} U_{0}\right)^{\prime}=\operatorname{tr} U_{0}^{\prime} U_{0}^{-1}=$ $\operatorname{tr}\left(U_{0}-K_{0} U_{0}^{-1}\right)$. We do not know though how to establish the integrability of In det $U_{0}$ by a geometric argument (a priori det $U_{0}$ may be arbitrarily small). In the case of a metric without conjugate points the formula $h_{\mu}=\int_{S M} \operatorname{tr} U d \mu$ can also be obtained by our method once Proposition 2.1 is established. In this case $r_{1}=\operatorname{tr} U$ may be negative but it is known to be bounded and hence integrable.

In estimating the entropy from below we will use

$$
h_{\mu}=\int_{S M} r_{3} d \mu
$$

and the following general fact. 
LEMMA 3.5. For all linear symmetric operators $U, M, N$, on a Euclidean space, if $U>0, M \geq 0$ and $N \geq 0$, then

$$
\operatorname{tr}\left(M U+N U^{-1}\right) \geq 2 \operatorname{tr} \sqrt{M} \sqrt{N}
$$

and the equality holds if and only if $\sqrt{M} U=\sqrt{N}$.

Proof. Let $S=\sqrt{M} \sqrt{U}-\sqrt{N} \sqrt{U^{-1}}$. We have tr $S S^{*} \geq 0$ and $\operatorname{tr} S S^{*}=0$ if and only if $S=0$. At the same time

$$
\begin{aligned}
\operatorname{tr} S S^{*} & =\operatorname{tr}\left(\sqrt{M} \sqrt{U}-\sqrt{N} \sqrt{U^{-1}}\right)\left(\sqrt{U} \sqrt{M}-\sqrt{U^{-1}} \sqrt{N}\right) \\
& =\operatorname{tr} \sqrt{M} U \sqrt{M}+\operatorname{tr} \sqrt{N} U^{-1} \sqrt{N}-\operatorname{tr} \sqrt{M} \sqrt{N}-\operatorname{tr} \sqrt{N} \sqrt{M} .
\end{aligned}
$$

Hence

$$
\operatorname{tr}\left(M U+N U^{-1}\right) \geq 2 \operatorname{tr} \sqrt{\mathrm{M}} \sqrt{\mathrm{N}}
$$

and the equality holds if and only if $\sqrt{M} \sqrt{U}=\sqrt{N} \sqrt{U^{-1}}$.

THEOREM 3.6.

$$
h_{\mu} \geq \int_{S M} \operatorname{tr} \sqrt{-K(v)} d \mu(v),
$$

and the equality holds if and only if the manifold $M$ is locally symmetric.

Proof. By Lemma 3.5

$$
h_{\mu}=\int_{S M} \frac{1}{2} \operatorname{tr}\left(U_{0}-K_{0} U_{0}^{-1}\right) d \mu \geq \int_{S M} \operatorname{tr} \sqrt{-K_{0}} d \mu=\int_{S M} \operatorname{tr} \sqrt{-K} d \mu .
$$

The equality holds if and only if $U_{0}(v)=\sqrt{-K_{0}(v)}$ for almost all $v \in S M$. Then for almost all $v \in S M, U(v)^{2}=-K(v)$. By continuity we have the last equality for all $v \in S M$. The Riccati equation (7) implies that $U^{\prime}=0$ on $S M$. Hence $U^{2}$ and therefore also $K$ are parallel along geodesics. But if $K$ is parallel along geodesics the geodesic reflection in any point of $M$ is a (local) symmetry. Thus $M$ is locally symmetric.

Remark 3.7. In the proof we estimate the exponential rate of volume growth pointwise. With our choice of volume element this rate has by Lemma 3.5 a global minimum in an open set of Lagrangian subspaces graph $U, U>0$. So we could obtain our estimate without the construction of invertible $U_{0}$, but then we would be unable to establish when the equality holds. In [W] such a method was formulated in the case of discrete time symplectic systems and applied to estimating the measure theoretic entropy of the gas of hard spheres.

\section{Acknowledgments}

The second author is grateful to Manfred Denker of the Mathematical Institute of the University of Göttingen and SFB 170 for their warm hospitality and support during his visit in the summer of 1987 when the final version of the paper was written.

The authors wish to thank the referee and Keith Burns for valuable comments.

\section{REFERENCES}

[F-M] A. Freire \& R. Mañe. On the entropy of the geodesic flow in manifolds without conjugate points. Invent. Math. 69 (1982), 375-392. 
[Os] V. I. Oseledets. A multiplicative ergodic theorem. Lyapunov characteristic numbers for dynamical systems. Trans. Moscow Math. Soc. 19 (1968), 197-231.

[O-S] R. Osserman \& P. Sarnak. A new curvature invariant and entropy of geodesic flows. Invent. Math. 77 (1984), 455-462.

[P1] Ya. B. Pesin. Characteristic Lyapunov exponents and smooth ergodic theory. Russ. Math. Surveys 32:4 (1977), 55-114.

[P2] Ya. B. Pesin. Formulas for the entropy of a geodesic flow on a compact Riemannian manifold without conjugate points. Math. Notes 24 (1978), 796-805.

[W] M. P. Wojtkowski. Measure theoretic entropy of the system of hard spheres. Ergod. Th. \& Dynam. Sys. 8 (1988), 133-153. 